\title{
MONEDA, DESARROLLO Y PENSAMIENTO ECONÓMICO LATINOAMERICANO: LECTURAS HETERODOXAS
}

\author{
MONEY, DEVELPOMENT AND LATIN AMERICAN ECONOMIC \\ THOUGTH: HETERODOX READINGS ${ }^{(*)}$
}

\author{
Monika Meireles $^{(* *)}$ \\ Universidad Nacional Autónoma de México, México (DC), México
}

Resumen: Este trabajo aborda el tema de la moneda y del crédito en la acumulación del capital a partir de distintos autores del campo heterodoxo del análisis económico, poniendo énfasis en sus diferencias teóricas con lo más comúnmente defendido por la ortodoxia económica. Ese balance fue hecho buscando los elementos trabajados por el poskeynesianismo radical, específicamente en lo que dice respecto a la Teoría Moderna del Dinero Endógeno, que funjan como inspiración en la discusión actual sobre la moneda y el desarrollo en el pensamiento económico latinoamericano. El ejercicio de fusionar teóricamente las dos tradiciones constituye parte esencial de estas "lecturas".

Palabras clave: Moneda; Desarrollo; Pensamiento económico latino-americano.

Abstract: This article aims to discuss how money and credit influenced capital accumulation. We took into consideration the contribution of authors in the heterodox field of economics, aiming to highlight the theoretical differences between their approach and the most common believes addressed by the orthodox stream. This effort was made searching for some elements worked by the radical poskeynesianism, mainly the Modern Money Theory, which can be used as inspiration in the current discussion about money and development in the Latin American economic thought. This theoretical exercise of merging two traditions is the essential part of these "readings".

Keywords: Money; Development; Latin-American economic thought.

(*) La autora agradece al Proyecto de Investigación e Innovación Tecnológica (PAPIIT IN301015), “Competencia Financiera Global y Regional: Modelos de Financiamiento Post-Crisis”, financiado por la DGAPA-UNAM.

$(* *)$ Doctora, Profesora de la Facultad de Economía la Universidad Nacional Autónoma de México (UNAM). E-mail: <meireles@usp.br>. Recibido en: 09.04.2015, aceptado en: 26.06.2015. 


\section{INTRODUCCIÓN}

El pensamiento económico latinoamericano tiene un timbre de voz propio, lo que no significa desarticulación entre su discurso y las preocupaciones investigativas de la agenda de trabajo de economistas heterodoxos de otras latitudes. La originalidad de su argumento no reside únicamente de la agudeza de la interpretación de la ciencia social nacida en el ámbito regional, ella más bien se ubica en el ingenio con el cual la mirada y la labor del intelectual latinoamericano se enriquece de la discusión llevada a cabo a nivel mundial. Así, no es nueva la preocupación que en la agenda de trabajo de los economistas latinoamericanos esté contemplada la revisión del debate de las corrientes heterodoxas en general, pero sin duda es bienvenida la noticia que recientemente la (CEPAL, 2015) haya reelaborado la invitación al baile entre los autores de "acá" y los de "allá", del cual mutuamente se ven nutridos tanto el neoestructuralismo como las aportaciones de la heterodoxia entendida de forma más amplia.

En esa tónica, y siguiendo los pasos dados por muchos de los autores que hacen parte del "Grupo UNAM" (GU), entendemos que de la fusión selectiva entre debates trabados alrededor de la Teoría Moderna del Dinero Endógeno del poskeynesianismo radical con las preocupaciones contemporáneas sobre el desarrollo en el pensamiento económico latinoamericano se puede acaparar una excelente posibilidad de enriquecimiento teórico en la trayectoria de ambas tradiciones.

Un ejemplo de cómo el ejercicio de fusión selectiva de dos paradigmas, como lo que aquí se propone, es muy fructífero fue dado por el rescate de los conceptos de soberanía monetaria y de banco central como prestamista y empleador de última instancia - tan caros a la teoría del dinero endógeno - como base para una discusión crítica de los limitantes que el mantenimiento de la dolarización formal impone al modelo alternativo de desarrollo encampado por el gobierno progresista de Ecuador bajo el mandato de Rafael Correa (MEIRELES, 2014). Sin embargo, si esa propuesta de acercamiento teórico entre tradiciones distintas repunta en nuestras ambiciones como un puerto de llegada seguro, el camino a navegar para su justificación definitiva es un poco más tormentoso, dado que su construcción es un proceso en marcha. Así, en el presente trabajo buscamos presentar brevemente algunas reflexiones sobre el matriz teórico de la construcción del entendimiento del fenómeno monetario en el poskeynesianismo radical, de tal forma que se trata más de un "mapa de estudio" de los autores que nos interesan mayormente, no constituyendo, por el momento, en una conclusión cabal sobre todas las temáticas que serían finalmente filtradas a partir de su abrazo con el pensamiento económico latinoamericano. En ese sentido, el texto se acerca más a una inspección intencionada en las aguas de la discusión sobre la moneda en clave heterodoxa para asegurar cuales son los canales más fácilmente navegables por parte de los teóricos inmiscuidos en el debate sobre el desarrollo latinoamericano actual.

El presente trabajo está dividido en tres partes que siguen a esa introducción. En la primera parte se discute a brocha gorda sobre como la neutralidad o no de la moneda fue abordada a lo largo de la historia del pensamiento económico. En la segunda parte se hace una revisión más detenida de los preceptos de la teoría monetaria poskeynesiana en contraposición con el entendimiento sobre la naturaleza del dinero ofrecida por 
el mainstream. Finalmente, en las conclusiones, se presentan algunos caminos para un diálogo más intenso entre heterodoxias: la representada por el dinero endógeno y la de las ciencias sociales latinoamericanas preocupadas por caracterizar al (sub)desarrollo y a la dependencia en el siglo XXI.

\section{LECTURAS SOBRE LA MONEDA: ORTODOXOS Y HETERODOXOS (PRIMER ROUND)}

La reflexión sobre la moneda, su naturaleza y funciones en la organización de la economía, precede en mucho el propio nacimiento formal de la ciencia económica - y es, sin duda, uno de sus objetos de estudio más escudriñados. En la tradición occidental, ya entre los antiguos griegos se interrogaban sobre su naturaleza y función - bien como sobre los desdoblamientos morales de una sociedad guiada por transacciones monetarias. Sin embargo, fue en el marco del liberalismo, con David Hume en el Siglo XVIII, que estas reflexiones se condensaron y dieron lugar a una explicación del funcionamiento de la demanda y de la circulación de la moneda a partir de lo que se convirtió posteriormente en la Teoría Cuantitativa de la Moneda (TCM), en la cual las decisiones de producción a corto plazo no son afectadas por la masa monetaria en circulación. De manera aún más sintética (INGHAN, 2004, p. 6), rescatando a Schumpeter, es categórico al afirmar que solo hay dos interpretaciones sobre la naturaleza del dinero que son relevantes: la que lo entiende como mercancía y la que lo ve como deuda o crédito. Es más común encontrar la primera interpretación en los análisis económicos de cuño ortodoxo, por su lado, en la heterodoxia hay una preferencia por entender al dinero poniendo más atención a su función como unidad de cuenta. Además, cabe destacar que en este lado del espectro de la interpretación, en el cual el "tiempo" y la "dinámica" son variables esenciales en la comprensión del fenómeno económico - por lo tanto más relevantes que el estudio de los "puntos equilibrio" y la forma de alcanzarlos - la importancia de la moneda y del crédito en el estudio de la acumulación del capital son completamente resignificados.

De manera general, vemos que uno de los debates más acalorados y fructíferos entre ortodoxos y heterodoxos se da alrededor de la creencia o no en la neutralidad de la moneda. Vale decir que no raras veces autores que se posicionan en el mismo lado de la diáspora no están completamente de acuerdo en las explicaciones de su origen e implicaciones. Una ilustración de lo afirmado es lo que ocurre en el seno de la tradición keynesiana. Hay un sustrato común de hipótesis que vinculan los herederos del profesor de Cambridge, sin embargo, en lo dice respecto al entendimiento de la cuestión monetaria y sus conexiones con la economía real y el ciclo económico las divergencias llevan a una tendencia explícitamente fratricida.

Se puede resumir, al interior de la heterodoxia, la polémica da el tono en la disputa por el legado de Keynes: poskeynesianos, y su creencia en la endogeneidad del dinero; versus neokeynesianos ${ }^{(1)}$, y su dislocada simpatía por la neutralidad y exogeneidad de la

(1) Elneokeynesianismo de la síntesis neoclásica está presente en seguidores de la microfundamentación de la macroeconomía keynesiana y de su armonización parcial con los modelos de equilibrio general de la teoría neoclásica. Entre los textos 
oferta de moneda. Además de abogar en pro de la neutralidad del dinero, estos economistas barrieron de sus análisis el hallazgo clave de Keynes: el rol de la incertidumbre en la economía y sus efectos en las decisiones intertemporales de los agentes económicos. A estos keynesianos ortodoxos o neokeyensianos, por su inconsistente sintonía con aspectos del mainstream que también se los hace clasificar como miembros de la sintesis neoclásica, (ROBINSON, 1976), les apodó con el inmejorable término de "keynesianismo bastardo". Ya para (DAVIDSON, 1992; 1982, p. 66), se trata de asociar esa corriente de rescate altamente cuestionable del keynesianismo con la figura literaria del gato de Alicia de Lewis Carroll, o sea, con nada más la manifestación espectral de una sonrisa burlona, pero padeciendo de la total ausencia de un cuerpo, en el caso entendido como una estructura claramente fiel a los preceptos keynesianos. Para la defensa de la perspectiva neokeynesiana y un ejemplo de la concepción teórica y pronóstico de política monetaria bajo la egida más moderna y elaborada de ese abordaje, véase (CLARIDA, 1999).

Otro punto que separa fundamentalmente a la perspectiva ortodoxa de la heterodoxa se asienta en la relaciones entre moneda y Estado. En un extremo podemos encontrar a la utopía hayekiana del dinero privado, en el cual el banco privado emisor del título más universalmente aceptado por el mercado gozaría del monopolio de emisión de la "moneda" y se convertiría así en su único y supremo gestor. De otro lado, evidentemente de los heterodoxos, es común encontrar la posición de los defensores de la regla tácita sobre la modernidad: "un Estado, una moneda". Avalando la sensible percepción no sólo de que el signo monetario tiene un rol preponderante en la psique de constitución de la soberanía de un pueblo, como adjudicando también a la soberanía monetaria su protagonismo en la constitución del espacio económico nacional.

Por su vez, en la teoría marxista, el tema monetario nutre también acalorado debate, aunque con enfoques y preocupaciones bastante distintos - pero sigue la tensión entre las interpretaciones que entienden la moneda como mercancía y la moneda como crédito-deuda encuentren una reedición en clave de teoría del valor -. Un ejemplo de las divergencias encontradas en el interior mismo de la corriente marxista que se dedica a estudiar la naturaleza del dinero como fenómeno social tiene raíz en los años setenta y se extiende hasta contemporáneamente. Es a partir de ahí que podemos destacar la siguiente polémica: la lectura althusseriana, de Suzanne de Brunhoff, que choca de frente con las concepciones que tienen por base una relectura más fina de la implicaciones filosóficas de la dialéctica marxiana, como es manifiesto en las obras de Ruy Fausto y Leda Paulani ${ }^{(2)}$. Además de la disputa entre una lectura más estructuralista y otra tributaria de una interpretación más a fin con el método dialéctico - incluso rescatando

precursores de esa tendencia se encuentra el manual Economics, de 1973, de Paul Samuelson, que tanto impacto tanto en Estados Unidos como en otras latitudes. Además, ese estilo de rescate simplista y deturpado de la macroeconomía keyenesiana hoy día todavía es ampliamente difundido, al menos a nivel de licenciatura, por la adopción casi que irrestricta del manual Macroeconomía, elaborado por Gregory Mankiw en los años noventa y que ya está en su sexta edición, tanto en español como en portugués.

(2) Eso sin mencionar a la interpretación también de cuño marxista llevada a cabo por autores como Fred Moseley, y sus alumnos en la Universidad de Massachusetts, campus de Amrherest, y Duncan Foley, en la New School, que no están directamente alineadas con ninguno de los entendimientos anteriores al respecto de la interpretación marxista de la moneda. 
mucho del joven Marx en su apreciación - encontramos a los circuitistas, precursores de la Teoría del Circuito Monetario en Francia, que fusionan al arsenal teórico que explica a la creación y destrucción del dinero en el circuito de acumulación capitalista elementos marxistas con bastante simpatía a algunas preocupaciones típicas keynesianas. De hecho, la reflexión sobre el modo de producción monetario en la obra de (PARGUEZ, 2006), uno de los principales exponentes del abordaje circuitista, es otra categoría que ofrece prometedor insumo a la actualización de la discusión sobre las características del subdesarrollo latinoamericano actual.

De esta breve muestra es fácil inferir que el debate al respecto de la naturaleza de la moneda y su rol en una economía capitalista-monetaria difícilmente genera consenso en el seno de una misma filiación teórica, que se dirá de los debates y disputas entre distintas corrientes. Así, hacer el recuento detallado de las corrientes y de cómo cada una entiende el fenómeno monetario extrapolaría, en mucho, el espacio que disponemos y desviaría el presente trabajo definitivamente de sus objetivos. Sin embargo, una breve revisión de algunas selectas aportaciones es fundamental, ya que lo que se produce sobre temas monetarios en la tradición de las ciencias sociales latinoamericanas resuena y también influencia - en menor o mayor grado - las posiciones y debates que se llevan a cabo en otras latitudes. Finalmente, cabe mencionar que diversos autores latinoamericanos, de manera no poco articulada con sus pares, sí se dedicaron - y siguen dedicándose - a la interpretación de los fenómenos de cuño monetario financieros de manera comunicante con el debate sobre el desarrollo de la región. Así, es importante reconocer que dentro de la tradición cepalina - tras toda la discusión sobre la inflación estructural de Noyola, Furtado y Sunkel - Maria da Conceição Tavares quizás sea el exponente más destacado de ese esfuerzo. Siguiendo dentro de la heterodoxia, los trabajos del uruguayo radicado en México, Samuel Lichtensztejn, siempre trataron de armonizar preceptos keynesianos y marxistas para mejor entender los fenómenos de la esfera del financiamiento al desarrollo en América Latina.

Hubo también importante contribución en el campo del marxismo latinoamericano, sobre todo en los años 60`s y 70's, siendo importante referencia el trabajo Ramón Ramírez y, posteriormente, de Juan Castaingts Teillery. Sin embargo, nos aflige constatar que sus trabajos no dieron lugar a la constitución de una agenda colectiva de investigación sobre la naturaleza de la moneda y su importancia en la problemática del 'capitalismo dependiente' que caracteriza la región.

\section{LECTURAS SOBRE LA MONEDA: ORTODOXOS Y HETERODOXOS (SEGUNDO ROUND)}

Entre las inúmeras formas de agrupación e identificación de las distintas interpretaciones de la historia del pensamiento económico y de la ciencia económica contemporánea hay una fisura metodológica básica que recurrentemente se encuentra en la literatura secundaria: la polarización entre el abordaje neoclásico y los múltiples enfoques que componen el abordaje heterodoxo. Ocioso resaltar que la escuela de pensamiento que domina la enseñanza y formación de los futuros profesionales de la carrera tiene raíz en el primero enfoque señalado. Sin embargo, es con especial regocijo que ya se hace notar 
actualmente un movimiento creciente en el sentido de obras de difusión que adoptan un herramental teórico heterodoxo como línea maestra de su interpretación.

El colorido abanico de interpretaciones dentro de la llamada "heterodoxia económica" es conformado por, entre otros, la paleta cromática abarca de marxistas a schumpeterianos, pasando por el regulacionismo francés, los institucionalistas, las economistas feministas, entre otros. El trazo en común que guardan es su herreña oposición a los planteamientos de la teoría neoclásica. Si la rivalidad es la marca entre las posiciones defendidas dentro de ese campo - tenida como estrategia para autodefinirse en oposición a la corriente competidora - también se encuentra, aunque marginalmente, autores que buscan puntos de intersección y cercanía en la agenda de investigación de su propia escuela con las demás, como es el caso entra la fusión en la heterodoxia estadunidense entre poskeynesianos y economistas radicales.

¿Pero cuáles son las características de la teoría económica heterodoxa que la diferencian de la economía ortodoxa? Cuatro son los aspectos metodológicos que marcan definitivamente la distinción entre uno y otro abordaje: a) epistemología; b) ontología; c) racionalidad; y d) centro del análisis. En cada uno de los campos señalados, en entendimiento de ortodoxia y heterodoxia son diametralmente opuestos. La ortodoxia, cimentada en el abordaje de la teoría neoclásica, se funda en: a) la creencia en el instrumentalismo científico, en lo cual la hipótesis de trabajo permite hacerse casi "infalibles" previsiones y puede guiar lo que sería la posición de equilibrio adecuada; b) el individualismo metodológico, que ultra-simplifica las relaciones sociales en las decisiones de un "agente soberano", el individuo, que en sus decisiones busca, siempre, maximizar su bienestar; c) la racionalidad substantiva, asegura que cualquier tomada de decisión por parte del individuo es hecha siempre con total conocimiento (híper-racionalidad) de los efectos económicos que esa acarrea; d) dominancia del análisis en el intercambio y en la obsesión con la escasez, haciendo con que sus modelos siempre se pauten por la decisión eficiente asignación de los recursos; y e) supremacía del mercado, como locus máximo, cuando operado libremente, de garantizarse el óptimo funcionamiento de la economía. La heterodoxia, por su vez, se yergue sobre la siguiente base metodológica: a) creencia en el realismo como matriz epistémica, entendido como primacía de la historia y sus hechos estilizados para la elaboración teórica; b) concepción organicista del patrón de decisión de los agentes económicos, las decisiones son marcadas por aspectos inherentes a las instituciones sociales; c) la racionalidad procedimental, que versa sobre las serias limitaciones que el individuo se encuentra en términos de conocimiento e información antes de su tomada de decisión; d) dominancia de aspectos referentes a la producción y la ganancia en el centro de los análisis; y e) la duda sobre la capacidad del marcado en su ropaje liberal fungir como responsable por la designación óptima de recursos, lo que justifica su constante necesidad de regulación por parte del Estado (LAVOIE, 2006, pp. 6-18).

De esa irreconciliable diferencia metodológica nace una implicación fundamental en términos de elaboración de sugerencias de política económica. De un lado, el Estado y su intervención en la vida económica es interpretado como el elemento responsable por las principales distorsiones que llevan al mal funcionamiento del mercado. La hete- 
rodoxia pone al revés la inferencia: el Estado es absolutamente esencial para regular la actividad económica y garantizar el mejor funcionamiento de los mercados.

En el campo de los estudios monetarios se puede ver de manera aún más clara como esa diferencia metodológica conlleva a resultados opuestos, tanto en lo que dice respecto al diagnóstico teórico como a las proposiciones de política económica. Sin remontar a la polémica entre bulionistas y anti-bullionistas, y el posterior debate entre defensores de la Currency y los de la Banking School, ambos en Inglaterra - tampoco sin profundizar sobre las posturas de los "metalistas" y sus contrincantes "papelistas" en el acalorado debate referente a mantenerse o no el patrón oro a fines del siglo XIX en América Latina( ${ }^{(3)}$ - podemos ilustrar las principales divergencias de ortodoxia y heterodoxia comparando la posición neomonetarista y la poskeynesiana.

La postura neomonetarista inspirada mayormente en los trabajos empíricos de Friedman y Anna Schwartz no son la única corriente de interpretación de teoría monetaria atacada por los poskeynesianos. Lo que se conoce como "síntesis neoclásica" o "síntesis keynesianas", que se formó en la lectura microfundamentada de Keynes por Hicks, Samuelson, Tobin y otros, difundida a través del modelo IS-LM de equilibrio combinado del mercado monetario y de financiamiento a la inversión, son igualmente cuestionados por configuraren una perturbación irreversible de los principales hallazgos teóricos de la revolución keynesiana, como es caso de la sumaria eliminación del rol de la incertidumbre en el modelo. Según (LAVOIE, 2006, p. 56), diez son las características que separan el entendimiento sobre el dinero del abordaje poskeynesiano del neomonetarista. El primero y fundamental punto de discordia es la percepción de que el dinero importa y tiene consecuencias en la economía y su oferta tiene forzosamente una contrapartida, para los adeptos a la visión poskeyensiana, mientras que los monetaristas lo ven como algo tan ajeno y unidireccional que pudiera haber sido "arrojado de un helicóptero", en lo quizás haya sido la metáfora aérea más famosa en las ciencias económicas y fue acuñada por Milton Friedman.

Además, los poskeyenesianos, por (LAVOIE, 2006), entienden el dinero como: a) una variable ora de flujo y ora de estoque; b) que su entrada en la economía se da a través de la producción; c) la oferta de dinero es endógena (ver apéndice 01); d) el crédito crea el depósito del público; e) la tasa de interés tiene claro impacto en la distribución del ingreso; f) la tasa de interés es fijada de manera exógena por el Banco Central (ver apéndice 02); g) la política monetaria restrictiva tiene impacto negativo en el aumento del producto tanto a corto como a largo plazo; h) no existe la "tasa natural" de interés; i) la velocidad de circulación de la moneda no es constante; j) la escasez de crédito se debe a un escenario de "falta de confianza" (4); y l) política monetaria y fiscal son ricos

(3) Para profundizar sobre el debate entre metalistas e papelistas en Brasil, en la segunda mitad del siglo XIX, entendido como un primero acercamiento del pensamiento económico latinoamericano sobra la neutralidad o no de la moneda y, incluso, defendiendo la idea de que la posición pro-crecimiento de los papelistas en esta polémica estuvo a raíz de los planteamientos desarrollistas de Vargas en el siglo XX, se recomienda el trabajo de (FONSECA; ROLLEMBERG, 2011).

(4) (WRAY, 2006, p. 59) hace una crítica acertada a lo que es un énfasis exagerado, por parte de la tradición poskeynesiana, en el aspecto de la incertidumbre en la demanda de dinero elaborado por Keynes. Para el autor, la demanda y atesora- 
instrumentos anti-cíclicos, siendo la última el estímulo más eficiente. Por su vez, el dinero en el abordaje neoclásico se presenta como: a) una variable de estoque (mensurable); b) teniendo su entrada en la economía a partir del intercambio; c) su oferta es exógena, lo que equivale a decir que es dependiente de la decisión de ofertar circulante por parte del Banco Central; d) el depósito del público en los bancos comerciales generan los recursos que serán usados para los préstamos futuros; e) la tasa de interés es resultado de las fuerzas del mercado; f) el nivel de la tasa de interés es producto del equilibrio entre oferta y demanda en el mercado monetario; g) la política monetaria sí puede tener efecto negativo en el nivel del ingreso de la economía, pero únicamente a corto plazo, pues a largo plazo las variables se ajustan a un nuevo y superior punto de equilibrio; h) la "tasa natural" de interés es la única que equilibra el mercado (variaciones alrededor de ella son temporarias); i) la velocidad de circulación de la moneda es constante; j) la escasez de crédito se debe a la falla conocida como "información asimétrica" en el mercado de fondos; y l) la política monetaria es la única eficaz para la corrección de los niveles de precios, siendo que esa debe obedecer la regla de aumento/disminución de la oferta monetaria por parte del Banco Central siguiendo el número de transacciones de la economía; la política fiscal es inocuo sobre el nivel de ingreso, por lo tanto, tiene carácter únicamente inflacionario.

La teoría monetaria poskeyenesiana se basa, así, en distintas primicias, siendo la principal la teoría del dinero endógeno, entendida como la negación de que la oferta de dinero pueda ser determinada arbitrariamente por la autoridad monetaria, o sea, que el volumen de la oferta monetaria se determina por la demanda de crédito bancario, y que esa, por su vez, es establecida en función de la demanda por dinero de los agentes. Vale mencionar que Keynes, además de reconocer el motivo demanda de dinero para transacciones comerciales, introdujo la identificación de la demanda por dinero por el motivo especulativo (vinculado al comportamiento de la tasa de interés y la intención del público en preservar su poder adquisitivo al largo del tiempo).

Bajo ese marco, tenemos que el rol del banco central va mucho más allá del bien comportado "guardián del valor de la moneda" a través del control de la base monetaria $^{(5)}$. Para la mayoría de los autores de esa tradición, su gestión debería concentrarse en tres aspectos fundamentales: a) proveer acceso a la liquidez en el mercado monetario a

miento de dinero motivada para aminorar la incerteza vinculada a la imprevisibilidad del escenario económico futuro es válido, legítimo, y una importante aportación de la corriente keynesiana. No obstante, le parece más promisor para el abordaje del dinero endógeno la retomada de otro aspecto también incorporado en el análisis por Keynes: el dinero como unidad de cuenta y el rol del Estado en el establecimiento de cuál será la moneda de cuenta nacional. Para tanto, propone que autores como Knapp y Lerner sean releídos con más atención, tanto en lo que dice respecto a su influencia en el pensamiento de Keynes sea adecuadamente mapeada, como para el perfeccionamiento continuo del abordaje.

(5) El control de la base monetaria que está implícito al manejo monetarista de la banca central - y que también es requisito para los regímenes de objetivo de inflación - es, en realidad, imposible. No solo a partir del abordaje del dinero endógeno se puede demostrar que la oferta de moneda no es una variable controlable por la autoridad monetaria. Algunos aportes contemporáneos del mainstream no son miopes para el hecho de que la capacidad creadora de crédito del sistema bancario inviabiliza la determinación de la base monetaria por parte del banco central. Sin embargo, lo que eses trabajos todavía no incorporan, es la noción de que el manejo de la política monetaria del banco central se concentra en la determinación de la tasa de interés, y de manera indirecta y bastante imprecisa, en el sustrato final de la liquidez en la economía. 
través de la disponibilidad de high-powered money en sus operaciones de open market; b) fungir como "prestamista de última instancia" (ver apéndice 03) e intentar asegurar que quiebras bancarias singulares no se conviertan en crisis financieras de mayores proporciones - o incluso en crisis económicas mayúsculas; c) supervisar y regular las actividades de los bancos y demás agentes privados del sistema financiero, con el fin de prevenir actividades ultraespeculativas que ponen en risco su solvencia y potencializan la vulnerabilidad del sistema como un todo; d) tener como objetivo fijar la tasa de interés a niveles que no penalicen la actividad productiva y el desarrollo; y e) tener en su horizonte de acción la maximización del empleo y del ingreso nacional, convirtiéndose en el "empleador de última instancia", o sea, que en momentos de baja del ciclo económico priorice medidas anticíclicas que estén comprometidas con la manutención - o recuperación - de los niveles de empleo.

\section{CONCLUSIONES}

En un texto reciente - envidiable por la profundidad y riqueza de detalles de su análisis - (PÉREZ; VERNENGO, 2012) argumentan acerca de la clara influencia del pensamiento de Keynes en la obra de Prebisch sobre las inconsistencias y fallas generadas cuando el mercado se autorregula y de cómo, a partir de esa contestación, la intervención del Estado con políticas proactivas y anticíclicas sería fundamental para atingirse un sistema económico más harmónico con los objetivos del desarrollo. Sin embargo, en el mismo estudio, se hace referencia a cómo las concordancias entre el economista argentino y el Lord inglés no significaron una adhesión total y acrítica de la influencia del segundo sobre el primero (ver apéndice 05). O sea, ya en esa "primera cita" entre la heterodoxia keynesiana y el pensamiento latinoamericano hubo roces importantes, como la ácida crítica de Prebisch a la supuesta carencia de adherencia a la realidad de algunas de las tesis presentadas en la Teoría General, sumado a la apreciación de que en dicho libro no se rompía completamente con la teoría "clásica" - como en el cuerpo del texto se llama a la teoría neoclásica -. Prebisch recurre al trabajo de Keynes en búsqueda de insights sobre la dinámica capitalista y el ciclo económico y en esa búsqueda se frustra pues esos no son los temas centrales tratados ahí por el economista de Cambridge (ver apéndice 04).

De esa anécdota emerge una importante enseñanza que se abre en par: a) al tenerse pertinente humildad intelectual y buscar en las teorías del "centro" elementos provechosos para ajustar el marco teórico propio - de tal forma que ese esté mejor aceitado para interpretar la especificidad de las economías latinoamericanas - no significa incurrir en "servilismo académico", "adulación eurocéntrica" o "malinchismo trasnochado"; y b) en el ejercicio de buscar inspiración en los debates trabados en otras latitudes, el latinoamericanista no está atentando en contra a la originalidad de sus planteamientos, más bien nutre sus argumentos con lo que considera más relevante en términos teóricos para seguir avanzando en su labor comprometido con la transformación de la sociedad en que está inmerso.

Finalmente, este trabajo estuvo dedicado a elaborar un breve rastreo sobre cómo el tema de la moneda y del crédito en la acumulación del capital fue abordado en distintos 
autores del campo heterodoxo del análisis económico. Ese balance fue hecho con un claro propósito: buscar los elementos trabajados por el poskeynesianismo radical, sobre todo en lo que dice respecto a la Teoría Moderna del Dinero Endógeno, que funjan como inspiración en la discusión actual sobre la moneda y el desarrollo en el pensamiento económico latinoamericano. En síntesis, podemos señalar que el pensamiento económico latinoamericano se enriquece a partir de la exploración de algunos de los argumentos de la vertiente poskeynesiana, a empezar por su atinada valorización del debate sobre la soberanía monetaria y su importancia para que se tenga un banco central con rol activo como prestamista y empleador de última instancia del sistema. El ejercicio de fusionar teóricamente parte de los elementos del poskeynesinanismo con los de los clásicos de las ciencias sociales latinoamericanas sobre el desarrollo y la dependencia ya arrancó, es un proceso en marcha. Hemos aquí identificado algunos de los grupos de trabajo que siguen por ese camino, como es el caso del Grupo UNAM, principalmente preocupados con las alternativas de desarrollo que emergen en un contexto de globalización financiera y que todavía no digiere los efectos de la crisis del 2007/2008.

\section{BIBLIOGRAFÍA}

ARESTIS, Philip. Post-keynesianism: a new approach to economics, Review of Social Economy, vol. 48, n. 3, 1990.

CHICK, Victoria. Is there a case for post keynesian economics? Scottish Journal of Political Economy, Vol. 42, n. 1, February, 1995.

CEPAL. Neoestructuralismo y corrientes heterodoxas en América Latina y el Caribe a inicios del siglo XXI. Libros de la CEPAL, n. 132 (LC/G.2633-P), Santiago de Chile, Comisión Económica para América Latina y el Caribe (CEPAL), 2015.

CLARIDA, Richard; GALÍ,Jordi; y GERTLER, Mark. The Science of Monetary Policy: A New Keynesian Perspective, Journal of Economic Literature, vol. XXXVII, December, 1999.

DAVIDSON, Paul. International Money and the real world. St. Martin's Press, New York, Davidson, 1992;1982.

FONSECA, Pedro y MOLLO, Maria. Metalistas x Papelistas: origens teóricos y antecedentes do debate sobre monetaristas e desenvolvimentistas. Serie Textos para Discussão, n. 348, UNB, 2011.

INGHAM, Geoffrey. The nature of money. Polity Press, Great Britain, 2004.

KALDOR, Nicholas. The Scourge of Monetarism. Oxford, Oxford University Press, 1985.

KING, John. E. Una historia de la economía poskeynesiana desde 1936. Akal, Madrid, 2009.

KNAPP, Georg. The State Theory of Money. Augustus M. Kelley, New York, 1973.

LAVOIE, Marc. Introduction to Post-Keynesian Economics. Palgrave Macmillan, Great Britain, 2006.

MEIRELES, Monika. Soberanía Monetaria y Alternativas de Desarrollo en Ecuador desde el Pensamiento Económico Latinoamericano. Tesis de doctorado, Programa de Posgrado en Estudios Latinoamericanos, UNAM, 2014.

PARGUEZ, Alain. Moneda y capitalismo: Lateoría general del circuito. En: Confrontaciones monetarias: marxistas y post-keynesianos en América Latina. Clacso, Buenos Aires, 2006.

PÉREZ, Esteban y VERNENGO, Matias. ¿Una pareja dispareja? Prebisch, Keynes y la dinámica capitalista. En: Estudios críticos del desarrollo, vol. II, núm. 3, segundo semestre de 2012. 
ROCHON, Louis-Philippe; SETTERFIELD, Mark. Post Keynesian interest rate rules and macroeconomic performance: a comparative evaluation. Paper presented at the Eastern Economic Association Annual Conference, 2007.

WRAY, Randall. El enfoque poskeynesiano del dinero. In: PIÉGAY, P.; ROCHON, L. Teorias monetarias poskeynesianas. Akal, Madrid, 2006.

WRAY, Randall. Money and credit in capitalist economies: the endogenous money approach. Edward Elgar, Great Britain, 1990.

\section{APÉNDICE 1}

La matriz teórica que tomados conjuntamente conforman el abordaje del dinero endógeno poskeynesiano puede ser desmenuzado en cuatro concepciones fundamentales (WRAY, 1990, p. 10): a) el entendimiento original de Keynes que la principal función del dinero era figurar como "unidad de cuenta"; b) nuevamente Keynes, pero en su entendimiento de que el dinero, y sobre todo la demanda por dinero; sirve como un activo seguro para aminorar la exposición en un mundo marcado por la incertidumbre; c) la aportación de Minsky resaltando el dinero como "débito" utilizado para financiar posiciones en activos; y d) la acertiva de Lavoie, enfatizando el dinero como crédito que financia el flujo de gastos. En una definición sintética: "In the endogenous approach to money, money enters the economy during normal economic processes [...] Thus, money is not a variable which can be added to the theory when it becomes convenient, since money enters into the determination of the most important variables which theory attempts to analyze. [...] money is a unit of account which transfers purchasing power from the future to the present. Once created, money serves as a medium of payment, and as a store of value. In a capitalist system, the important debts are those which are created as capital assets are privately produced and purchased. These debts involve the creation of money, and money is used to retire the debts, which destroys money. Thus, money is endogenously created as assets are produced and financed, and is endogenously destroyed as positions are liquidated" (WRAY, 1990, pp. 72-73). Es interesante observar como en la última parte de su definición, extraída de su tesis doctoral, Wray se acerca a la teoría del circuito monetario, sin embargo, en sus escritos más actuales, su fundamentación de la endogeinidad del dinero se apoya en el enfoque chartalista o neo-chartalista, inspirado en la obra de Knapp.

\section{APÉNDICE 2}

Analizando la efectividad o no de la política monetaria en términos de elevación del producto, (ROCHON; SETTERFIELD, 2007) identifican dos vertientes dentro del poskeynesianismo: a) el abordaje activista, que defiende el uso de la tasa de interés como instrumento de política monetaria anticíclica, tratando de mejorar el nivel del producto y regular el ciclo económico (autores como Basil Moore y Thomas Palley son representantes de esa postura); y b) el grupo que defiende que la tasa de interés sea 'estacionada' a determinado nivel, pues autores como John Smithin, Marc Lavoie, Randall Wray, Mario Seccareccia y el propio Louis-Philippe Rochon, argumentan que la política monetaria no es un instrumento confiable para regular los niveles del producto agregado y siguen defendiendo que la po- 
lítica fiscal es aquella que debe ser utilizada para alcanzar los objetivos macroeconómicos deseados referentes al dinamismo de la actividad económica. Además, los autores señalan la existencia de tres subdivisiones interpretativas al interior de esa última vertiente: a) la que defiende la "regla de Smithin", entendiendo que la tasa real de interés deba ser significativamente baja o incluso cercana a cero; b) la trabajada en la Universidad de Missouri o "regla de Kansas City", que defiende que la tasa nominal de interés deba ser muy baja, cercana a cero o incluso capaz de generar tasas de interés real negativas; y c) la "regla de Pasinetti", defendiendo que la tasa de interés real debería ser igual a la tasa de crecimiento de la productividad del trabajo. Como recomendación de política, se debe llevar en cuenta el impacto distributivo distinto que cada una de las tres reglas de fijación de la tasa de interés de orientación poskeynesiana tiene, tratando de encontrar aquella que favorezca el objetivo de dinamismo económico junto con la estabilidad de precios.

\section{APÉNDICE 3}

Resaltando el aspecto de que la banca central acomoda enteramente el volumen de liquidez que es demandada por la economía - admitiendo, así, una curva de oferta de moneda horizontal - (KALDOR, 1985, p. 47) destaca la función de prestamista de última instancia como la principal desempeñada por la autoridad monetaria. De manera más detallada y en en sus palabras,

"the (Central) Bank cannot refuse the discounting of 'eligible bills' rendered to it by the discount houses. If it did, by setting a fixed limit to the amount which the (Central) Bank is prepared to discount on a daily or a weekly basis (in the same way as the box office of a theatre is willing to sell a fixed number of tickets for a performance) the (Central) Bank would fail in its function as 'lender of last resort' to the banking system which is essential to ensure that the clearing banks do not become insolvent as a result of lack of liquidity. Precisely because the monetary authorities cannot afford the disastrous consequences of a collapse of the banking system, while the banks in turn cannot allow themselves to get in a position of being 'fully stretched', the 'money supply' in a credit-money economy is endogenous, not exogenous - it varies in direct response to changes in the public 'demand' to hold cash and bank deposits and not independently of that demand".

Aunque (WRAY, 1990, p. 94) no comparta de la interpretación que defiende que la curva de oferta monetaria sería, en todos sus puntos, totalmente elástica en relación al nivel de la tasa de interés - o sea, horizontal - en su entendimiento Kaldor, en consonancia con Keynes, atina en resaltar el rol de prestamista de última instancia del banco central. De hecho, el economista de la Universidad de Missouri, destaca como los dos principales principios del abordaje del dinero endógeno: a) que la curva de oferta monetaria no puede ser independiente de la curva de demanda, justamente por el comportamiento interesado en obtener ganancias que dictamina el perfil de la actividad empresarial que demanda crédito para invertir; y b) la enseñanza histórica aprehendida a partir de la Grande Depresión y de las crisis financieras esporádicas desde entonces versa sobre la responsabilidad del banco central como fundamental garantizador de la estabilidad financiera, a través del mantenimiento de la liquidez y previniendo, así, el temido encadenamiento deuda-deflación. 


\section{APÉNDICE 4}

Como nos explican los autores: "Prebisch no encontraría en Keynes lo que buscaba. El objetivo de la teoría de Keynes (centraba el caso de una economía capitalista avanzada) era explicar las causas del aumento o disminución del empleo y la utilización de la capacidad ya existente. Ni el análisis del ciclo ni la dinámica constituían una preocupación central de la obra de Keynes, siendo la principal preocupación la demostración de la posibilidad de una posición de equilibrio con desempleo. Dicho en otras palabras, se trataba de demostrar que las economías de mercado tienden hacia posiciones de equilibrio con desempleo, incluso si se cumplieran todas las condiciones de una situación de competencia perfecta con plena flexibilidad de precios. La razón esencial para explicar la persistencia del desempleo radicaba en que las economías de mercado son, ante todo, economías monetarias, economías en las cuales el dinero juega un papel esencial, porque es el nexo entre el presente y un futuro incierto" (PÉREZ; VERNENGO, 2012, pp. 167-168).

\section{APÉNDICE 5}

Es así que podemos afirmar que "Keynes tuvo una profunda influencia en el pensamiento de Prebisch no sólo en términos del diagnóstico acerca de los principales fallos de las economías de mercado, sino también en la necesidad de llevar a cabo políticas anticíclicas. No obstante, el camino por el cual Prebisch desarrolló sus ideas no fue lineal, ni puede ser descrito como una aceptación acrítica de las ideas desarrolladas en los países centrales. De hecho, Prebisch fue un crítico bastante duro de la Teoría general. Prebisch sostuvo que la teoría de Keynes era inconsistente y no representaba en absoluto una ruptura con la lógica del análisis clásico. En particular, Prebisch correctamente nota que los argumentos centrales de la Teoría general son incapaces de escapar completamente de la visión imperfeccionista de los problemas del desempleo, en lo que tenía razón en última instancia, y a pesar del hecho de que Keynes también quería escapar de las explicaciones basadas en rigideces" (PÉREZ; VERNENGO, 2012, pp. 187-188). 